\title{
PERFORMANCE ANALYSIS OF A SECOND ORDER DELAY-LOCK LOOP WITH APPLICATION TO A CDMA SYSTEM WITH MULTIPATH PROPAGATION
}

\author{
J.J.Olmos, R.Agustr
}

Dept. de Teoria del Senyal i Comunicacions

Universitat Politècnica de Catalunya (UPC)

Apdo. 30.002, 08080 Barcelona, SPAIN

\begin{abstract}
CDMA systems require synchronization between the received sequence and the locally generated sequence. This is done by means of a Delay-Lock Loop (DLL). The differential equation of an incoherent secondorder DLL has been programmed. The Mean Time to Lose Lock (MTLL) of the DLL is a very important design parameter. We have obtained this parameter by computer simulation and we are able to plot the MTLL as a function of the signal to noise ratio in the data bandwidth. In a narrow-band system, our simulation shows that the effect of the fast Rayleigh fading is a remarkable performance degradation even with high mean signal to noise ratio. Assuming a wide-band system, so that the receiver has enough bandwidth to resolve the different propagation paths, we propose a modified DLL scheme. The MTLL of the proposed DLL is evaluated using a dynamic simulation of the multipath channel. The results show an improvement with respect to the conventional DLL.
\end{abstract}

\section{DLL DESCRIPTION}

Usually CDMA systems achieve synchronization between the local and the received sequences in two steps. First, a coarse alignment is performed by a code acquisition circuit, and then a DLL is commonly used to achieve and keep fine tracking of the received sequence phase. Normally, the low signal to noise ratio of the received CDMA signal prevents a proper carrier recovery. So, an incoherent DLL is of interest in this case.

The received CDMA signal can be written as,

$$
y(t)=\sqrt{2 P} b(t) a(t-\tau) \cos \left(\omega_{0} t+\theta\right)+n(t)
$$

where $a(t)$ is the transmitted PN sequence, $b(t)$ is the data sequence and $\omega_{0}$ and $\theta$ are, respectively, the carrier pulsation and phase. $a(t)$ takes on values from the set $\{ \pm 1\}$ each $T_{c}$ seconds (chip period), and $b(t)$ takes on values from the set $\{ \pm 1\}$ each $T$ seconds (bit period), where $T>T_{c^{*}} P$ is the received signal power and $\tau$ is the propagation delay to which the DLL must lock. $n(t)$ is white gaussian noise with spectral density $G_{n}(f)=N_{o} / 2$. The block diagram of an incoherent DLL

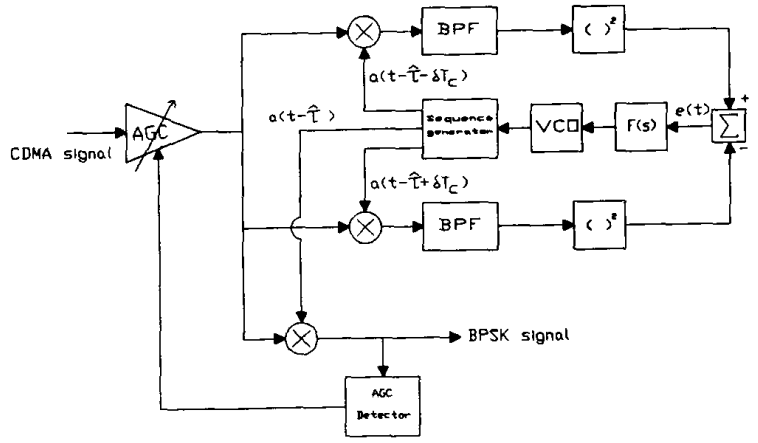

Figure 1: Block diagram of an incoherent DLL

is shown in figure 1 . In figure $1,2 \delta$ is the time shift between the early and late codes and the bandwidth of the band-pass filters, $B \approx 1 / T$ allows the filtering of the data signal without distortion. The behavior of the DLL can be described by a differential equation, [1]:

$$
\frac{d \epsilon}{d t}=\frac{1}{T_{c}} \frac{d \tau}{d t}-K P f(t) *(S(\epsilon, \delta)+N(t))
$$

In (2), $\epsilon \Delta(\tau-\hat{\tau}) / T_{c}$ is the shift between the local and the received sequence in chips, $d \tau / d t$ is the initial clock frequency offset, $K$ is the VCO constant, $f(t)$ is the low-pass filter impulse response, $S(\epsilon, \delta)$ is the " $S^{n}$-curve and $N(t)$ is noise with spectral density near the origin given by, [2]:

$$
G_{N}(0, \epsilon)=\frac{2 N_{0}}{P}\left[\lambda(\epsilon, \delta)+\frac{1-g^{2}(\delta)}{\gamma}\right)
$$

where $\gamma=P / N_{0} B$ is the signal to noise ratio in the data bandwidth, and

$$
\begin{aligned}
\lambda(\epsilon, \delta) \triangleq R_{a}^{2}\left[(\epsilon-\delta) T_{c}\right]+R_{a}^{2}\left[(\epsilon+\delta) T_{c}\right]-2 g(\delta) R_{a}\left[(\epsilon-\delta) T_{a}\right] R_{a}\left[(\epsilon+\delta) T_{c}\right] \\
g(\delta)=\left\{\begin{array}{cc}
1-2 \delta, & (0 \leq \delta \leq 0.5) \\
0, & (\delta>0.5)
\end{array}\right. \\
R_{a}(\epsilon)=\left\{\begin{array}{cc}
1-|\epsilon|, & (|\epsilon| \leq 1) \\
0, & (|\epsilon|>1)
\end{array}\right.
\end{aligned}
$$

08.04.1 


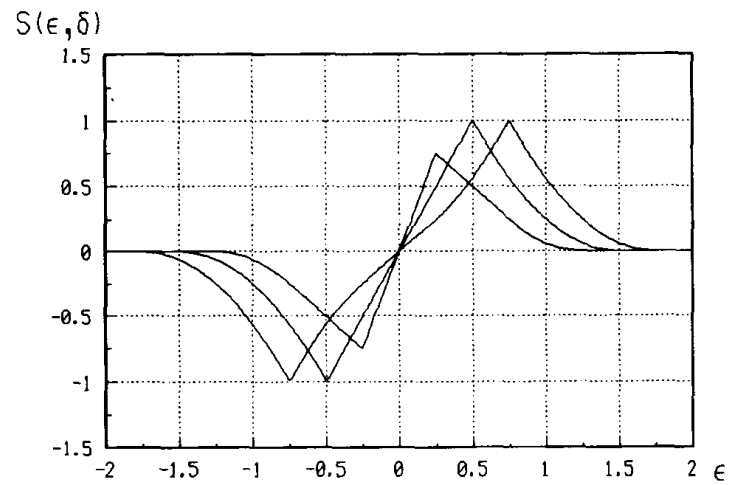

Figure 2: "S" curve for $\delta=0.25,0.50$ and 0.75 .

The loop " $S$ "-curve is shown in figure 2 . It can be shown that its slope at the origin is $4(1-\delta)$. As can be seen from (2), the closed loop gain of the DLL depends on the received signal power. In a narrow-band transmission, where the receiver can not resolve the different propagation paths, deep signal fading are likely to happen thus changing the DLL parameters. To avoid this we have assumed the use of an AGC circuit driven by the BPSK signal power. We assume that the AGC is fast enough in order to track the Rayleigh fading. A real AGC circuit keeps constant the power of the signal plus noise. So, if $\boldsymbol{P}_{\boldsymbol{o}}$ is the mean value of the received signal power, and $\gamma_{0}$ is the mean signal to noise ratio, after the AGC circuit we have at any moment, [1]:

$$
\frac{P}{P_{0}}=1+\frac{1}{\gamma_{0}} / 1+\frac{1}{\gamma}
$$

The second order DLL parameters, natural frequency, damping factor and equivalent noise bandwidth are, respectively:

$$
\omega_{n}=\sqrt{\frac{4(1-\delta) K P}{\tau_{1}}} ; \quad \xi=\frac{\omega_{n} \tau_{2}}{2} ; \quad B_{L}=\frac{\omega_{n}}{2}\left(\xi+\frac{1}{4 \xi}\right)
$$

where $\tau_{1}$ and $\tau_{2}$ are the time constants of the first order active loop filter. The loop filter is designed in order to achieve the desired DLL parameters when $P=P_{0}$ and $\gamma=\gamma_{0}$. As the AGC is not ideal, this parameters are subject to changes depending on the channel attenuation.

By computer simulation of the differential equation (2), we have found that in the presence of an initial clock frequency offset $t$ and in a noiseless situation, the lock-in of the DLL is not guaranteed unless $\tau / \omega_{n} \leq 2 \delta$. This is quite exact for $\delta<0.5$ and somewhat pessimistic for $\delta>0.5$. So, with an active loop filter and $\xi=0.7071$ there is a lower bound for the noise bandwidth of the loop given by:

$$
B_{L} \geq \frac{0.53 \cdot \Delta f_{v c o} \cdot R_{c}}{2 \delta}
$$

where $\Delta f_{V C o}$ is the VCO stability and $R_{c}$ is the sequence chip rate. Notice that $R_{c}$ can be expressed as $B$ times the processing gain $(P G)$.

As the loop "S"-curve is not periodic, due to the presence of noise the DLL will sooner or later lose lock. The Mean Time to Lose of Lock (MTLL) is a very important design parameter. We have obtained this magnitude, for the second order DLL, by computer simulation.

In section 2 of this paper we describe the computer simulation that has been carried out. In section 3 we show results for a static fading channel and for a narrow-band situation with fast changing Rayleigh fading. In section 4 we propose an alternate DLL design suitable for multipath propagation environment and assess its performance in a tworay channel.

\section{SIMULATION DESCRIPTION}

In order to simulate the DLL operation, the differential equation (2) has been translated into an incremental recursive equation and programmed. The time interval between the update of the variables is small compared to $B_{L}{ }^{-1}$, so results are quite precise. The noise process in (2) is modeled by uncorrelated samples of a computer generated gaussian process whose standard deviation depends on the instantaneous signal to noise ratio, $\gamma$. In a narrow-band transmission, $\gamma$ is obtained by multiplication of $\gamma_{0}$ (which is a data of the program) times the instantaneous channel attenuation.

We assume that the channel follows a Gaussian Wide-Sense Stationary Uncorrelated Scattering (GWSSUS) model. Up to five independent processes of complex white gaussian noise are generated by Montecarlo methods. The power spectrum of this processes, that model the different independent propagation paths, are independently shaped by digital filtering with third order Butterworth digital filters. The bandwidth of this filters can be adjusted to simulate different values of the "Doppler Spread" ( $\sigma$ ) of each path. The mean power of each propagation path and the relative time delay between them can also be adjusted to fit any given "Power Delay Profile". White gaussian noise is finally added to the received signal in order to simulate the receiver front-end noise.

To obtain an estimation of the MTLL of the system, we initialize to zero the alignment error $\epsilon$. Then, the channel and the DLL freely interact until $\epsilon$ gets out of the range of values for which the " $S$ " curve is not zero. This situation is considered as an "out-of-lock" situation and the DLL is then reset to $\epsilon=0$ and the DLL section of the program is stopped. Then we let the channel section of the program run alone until 
the channel attenuation for each path is equal $( \pm 0.5 d B)$ to its mean value. This situation is considered favorable for the coarse alignment circuit to recover lock, and so the DLL is started again and a new interaction with the channel begins. When 100 "out-of-lock" situations have been counted, the MTLL (expressed in bits) is approximated by the total number of bits processed divided by 100 .

\section{SIMULATION RESULTS ASSUMING A NARROW- BAND TRANSMISSION}

In this case only one propagation path has to be considered. So, deep fading of the received signal are likely to happen. To obtain the instantaneous channel attenuation we simply use the squared modulus of the first complex propagation path of our simulator. The probability density function of $\gamma$ is then $f_{\gamma}(\gamma)=\left(1 / \gamma_{\partial}\right) \exp \left(-\gamma / \gamma_{0}\right)$. To evaluate the influence of the Rayleigh fading, we first show results for a static fading channel. The considered DLL is designed to have $B_{L} / B=5.3 \cdot 10^{-4}$ for $\gamma_{0}=5 d B$ and $\delta=0.5$. The value for $B_{L}$ is the minimum allowable according to (7) for a product $\Delta f_{v C O} \cdot P G=10^{3}$. Figure 3 shows the obtained MTLL as a function of $\gamma$. We see that, even with a static fading of $15 \mathrm{~dB}$, the performance is quite good. In fact, with $B=10^{d} \mathrm{~Hz}$ $(10 \mathrm{Kbit} / \mathrm{sec})$, for example, we would have $M T L L=123$ minutes.

If for the same DLL, $\gamma$ is allowed to vary dynamically, being its mean value $\gamma_{0}=5 d B$, the performance is quite poorer. The results are shown in table I. In table I we see that when the "Delay Spread" of the channel is divided by ten, the MTLL is approximately multiplied by ten. But, in this case, to achieve a performance similar to that of the static channel the "Delay Spread" must be extremely low, which is unrealistic. For $B=10^{4} \mathrm{~Hz}$, for example, we need $\sigma=5.3 \cdot 10^{-4} \mathrm{~Hz}$.

Table I: MTLL with fast Rayleigh fading

\begin{tabular}{|c|c|}
\hline$\sigma / B$ & $\log (M T L L \cdot B)$ \\
\hline $5.3 \cdot 10^{4}$ & 4.55 \\
$5.3 \cdot 10^{5}$ & 5.20 \\
$5.3 \cdot 10^{6}$ & 6.03 \\
$5.3 \cdot 10^{7}$ & 6.90 \\
$5.3 \cdot 10^{8}$ & 7.64 \\
\hline
\end{tabular}

\section{DESCRIPTION AND PERFORMANCES OF THE PROPOSED DLL}

In a wide-band transmission, the receiver may have enough bandwidth to resolve more than one propagation path. In this situation we can make use of the fact that the signals arriving from different paths have been independently faded and so it

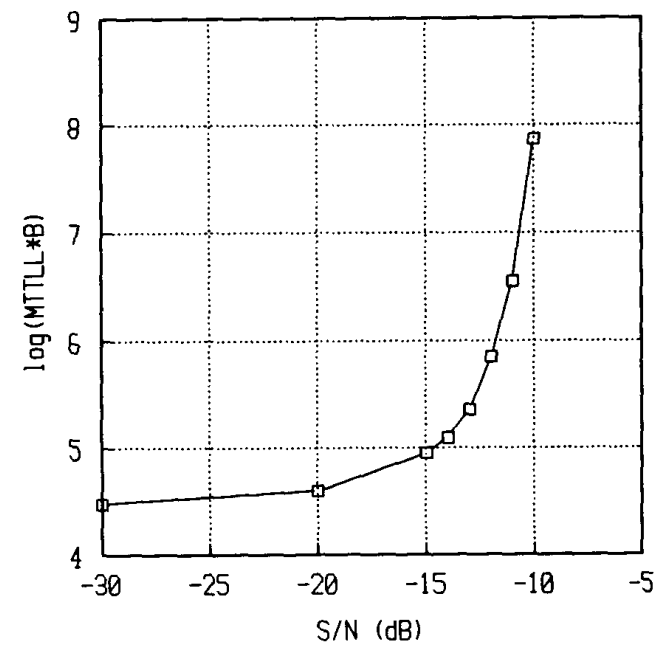

Figure 3: MTLL with a static fading channel

may be understood as a diversity system. In figure 4 we show the block diagram of the proposed DLL. In general, it may have up to $M$ branches, $M$ being the total number of propagation paths. For simplicity, the case $M=2$ has been plotted. Also, the AGC circuits (one for each branch) have been omitted. The coefficients $A_{i}(i=1, \ldots, M)$ are chosen to give more weight to the branches with the best signal to noise ratio.

The analysis of the proposed scheme lies on the fact that the noise processes at the output of each branch can be considered as approximately uncorrelated. The error signal in the $i$ branch can be written as:

$$
e_{i}(t)=P_{i} S(\epsilon, \delta)+N_{i}(t)
$$

where $P_{i}$ is the signal power in the $i$ branch and $N_{i}(t)$ the associated noise process. We assume that $\gamma_{l}$, the signal to noise ratio for the $i$ branch, has an exponential probability density function with a mean value given by $\gamma_{i, n o m}$. The new differential equation is:

$$
\begin{gathered}
\frac{d \epsilon}{d t}=\frac{1}{T_{c}} \frac{d \tau}{d t}-K P f(t) *\left[S(\epsilon, \delta)+\frac{\sum_{i=1}^{M} A_{i} N_{i}(t)}{P}\right] \\
{\left[P_{\Delta} \sum_{i=1}^{M} A_{i} P_{i}\right]}
\end{gathered}
$$

and the total noise spectral density near the origin is given by: 


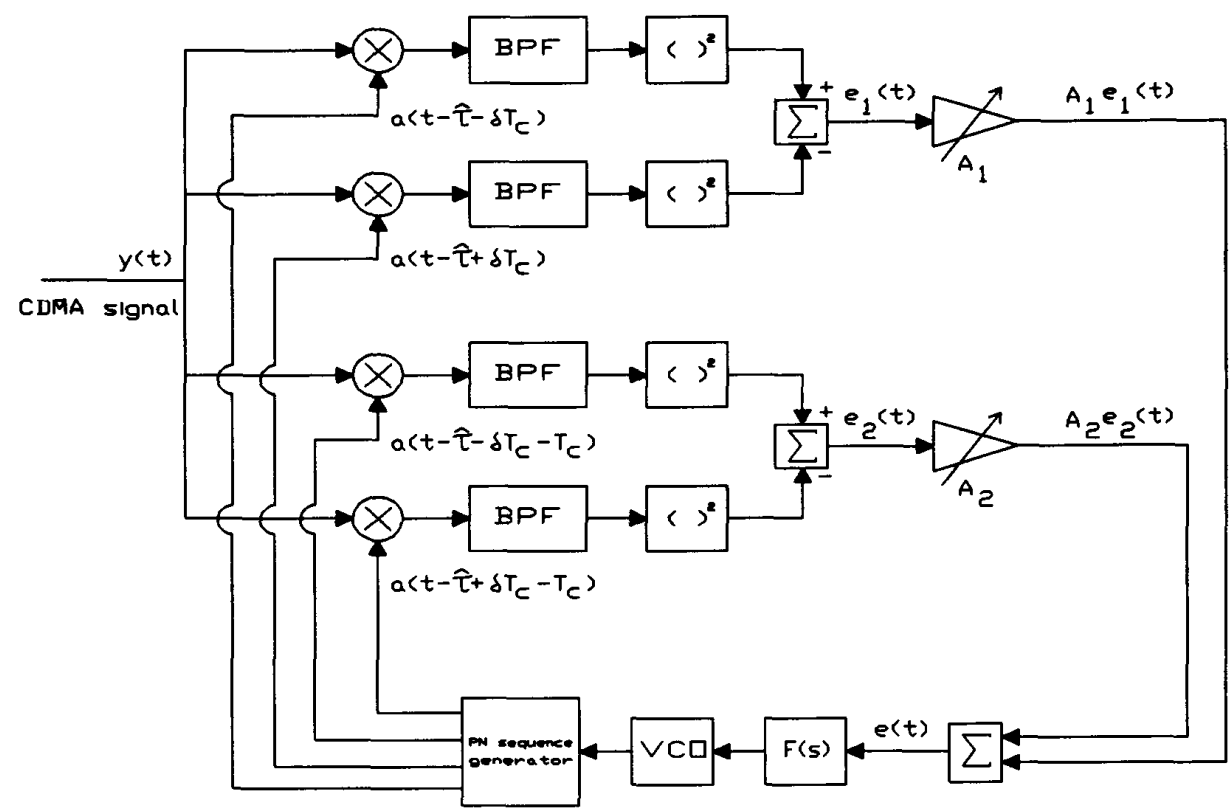

Figure 4: Proposed DLL block diagram

$$
\begin{aligned}
& G_{M}(0)=\frac{2}{B} \sum_{i=1}^{M}\left[\frac{A_{i} P_{i}}{P}\right]^{2} \frac{1}{\gamma_{i}}\left[\lambda(\epsilon, \delta)+\frac{1-g^{2}(\delta)}{\gamma_{i}}\right) \\
& \left(N(t) \Delta \frac{\sum_{i=1}^{M} A_{i} N_{i}(t)}{P}\right)
\end{aligned}
$$

We assume that when $\gamma_{i}=\gamma_{i, \text { nom }}(i=1, \ldots, M)$ the noise power is the same for all the branches. Then it can be shown that, at any time, the AGC circuits force the condition:

$$
\frac{P}{P_{i}}=\left[\frac{1+\frac{1}{\gamma_{i}}}{1+\gamma_{i, \text { mam }}}\right]\left(\sum_{j=1}^{N} A_{j} \frac{1+\gamma_{j, \text { nam }}}{1+\frac{1}{\gamma_{j}}}\right] \quad(i=1, \ldots, M)
$$

The expression (11) is used to evaluate (10) in order to find the instantaneous standard deviation of the computer generated noise samples. As with the conventional DLL, the design only guarantees the wanted DLL parameters $\left(B_{L}, \omega_{n}\right.$, and $\left.\xi\right)$ when $\gamma_{i}=\gamma_{1, \text { nom }}(i=1, \ldots, M)$.

\subsection{Simulation results}

In order to assess the performance of the proposed DLL we take a product $\Delta f_{v c o} \cdot P G=10^{3}$ and $\delta=0.5$. This implies a value for the DLL noise bandwidth of $B_{L} / B=5.3 \cdot 10^{4}$. The channel is considered to have two independent propagation paths with a relative delay of $T_{c}$ seconds. The mean signal to noise ratios, $\gamma_{1, \text { nom }}$ and $\gamma_{2, \text { nom }}$ are known. To see the influence of the second ray, we have taken $\gamma_{1 \text { rom }}=5 d B$ in all the cases and $\gamma_{2, \mathrm{nom}}$ growing from $\gamma_{2, \mathrm{nom}}=-15 d B$ to $\gamma_{2, \mathrm{nom}}=5 d B$ in steps of $5 d B$. In every case we have taken $A_{l}=1$ and $A_{2}=\gamma_{2, \mathrm{mom}} / \gamma_{1, \mathrm{mom}}$. The DLL is redesigned every time that $\gamma_{2, \text { nom }}$ is changed.

Table II: MTLL for the proposed DLL

\begin{tabular}{|c|c|c|}
\hline$\gamma_{2, \text { nam }}(d B)$ & $\begin{array}{c}\sigma / B=0.001 \\
\log (M T T L L\end{array}$ & $\begin{array}{c}\sigma / B=0.01 \\
\log (M T T L \cdot B)\end{array}$ \\
\hline-15 & 4.55 & 4.48 \\
\hline-10 & 4.66 & 4.58 \\
\hline-5 & 5.27 & 5.34 \\
\hline 0 & 6.3 & 6.45 \\
\hline 5 & 7.33 & 7.5 \\
\hline
\end{tabular}

For the "Doppler Spread" of the channel we have chosen two values: $\sigma / B=0.01$ and $\sigma / B=0.001$. This can model a "Doppler Spread" of $100 \mathrm{~Hz}$ with $B=10^{4} \mathrm{~Hz}$, in the first case, and with $B=10^{5} \mathrm{~Hz}$ in the second case. The simulation results are shown in table II. This results show a remarkable

\subsection{4}

0212 
improvement of the performance with respect to the conventional DLL. With $B=10^{4} \mathrm{~Hz}$, for example, and two paths of the same mean signal to noise ratio $(5 d B)$ the MTLL would be 48 minutes. Moreover, the "Doppler Spread" of the channel in this case $(100 \mathrm{~Hz})$ fits to a realistic situation. The value of $\sigma$ is not determinant for the overall performance since, like in a diversity system, the fluctuations of the overall signal to noise ratio are not as deep as for the conventional case. It can be noticed that for $\gamma_{2 \text {,nom }}=-15 d B$ the MTLL is the same that for the narrow-band system. This is what we should expect since, in this case, the contribution of the second ray is almost negligible. Though the impulse response of the channel could be known by the receiver, we have not taken into account the possibility that the weights $A_{i}$ could be adaptive. The fluctuations in this weights would force undesired changes in the closed loop gain of the DLL and in the DLL parameters.

\section{CONCLUSIONS}

The differential equation of an incoherent DLL has been programmed and the DLL performance has been tested in a dynamical fading situation. A channel simulator program, which allows the selection of the channel "Power Delay Profile" and "Doppler Spread", has been developed. In a narrow-band transmission with fast Rayleigh fading the obtained MTLL seems too low for normal applications. A new DLL scheme, with application to a multipath propagation situation, has been proposed and tested. The obtained performance shows an improvement with respect to the conventional DLL. The unavoidable condition required for the implementation of this scheme is that the receiver should have enough bandwidth to resolve more than one propagation path.

\section{REFERENCES}

[1] J.K.HOLMES, "Coherent Spread Spectrum Systems", John Wiley \& Sons, 1982

[2] A.POLYDOROS, C.L.WEBER, "Analysis and Optimization of Correlative Code-Tracking Loops in Spread-Spectrum Systems", IEEE Trans. on Comm. Vol COM-33, NO.1, January 1985.

08.04.5 